\title{
OPEN High speed, complex wavefront shaping using the digital micro-mirror device
}

\begin{abstract}
Ahmed B. Ayoub $₫$ \& Demetri Psaltis
Digital micro-mirror devices (DMDs) have been deployed in many optical applications. As compared to spatial light modulators (SLMs), they are characterized by their much faster refresh rates (full-frame refresh rates up to $32 \mathrm{kHz}$ for binary patterns) compared to $120 \mathrm{~Hz}$ for most liquid crystal SLMs. DMDs however can only display binary, unipolar patterns and utilize temporal modulation to represent with excellent accuracy multiple gray-levels in display applications. We used the built-in time domain dynamic range representation of the DMD to project 8-bit complex-fields. With this method, we demonstrated 8-bit complex field modulation with a frame time of $38.4 \mathrm{~ms}$ (around $0.15 \mathrm{~s}$ for the entire complex-field). We performed phase conjugation by compensating the distortions incurred due to propagation through free-space and a scattering medium. For faster modulation speed, an electrooptic modulator was used in synchronization with the DMD in an amplitude modulation mode to create grayscale patterns with frame rate $\sim 833 \mathrm{~Hz}$ with display time of only $1.2 \mathrm{~ms}$ instead of $38.4 \mathrm{~ms}$ for time multiplexing gaining a speed up by a factor of 32 .
\end{abstract}

SLMs have been widely used in optical applications including wave-front shaping and light focusing ${ }^{1-8}$. A main limitation of liquid crystal SLMs is their low refresh rates, typically less than $120 \mathrm{~Hz}$. This limitation can make it impossible to achieve high performance in applications that require high speed such as beam focusing in live samples. This has opened the door for another technology, DMDs, to be used for similar applications ${ }^{9-14}$. As opposed to SLMs, DMDs are characterized by their much faster refresh rates. DMD devices have a maximum refresh rate of $32 \mathrm{kHz}^{15}$. However, as an "on-off" device, DMD allows only binary amplitude-modulation as compared to the grayscale and phase modulation that is possible with SLMs. Despite its binary amplitudemodulation, it was shown that DMDs can outperform SLMs in beam-shaping applications ${ }^{16}$. $\operatorname{In}^{17}$, Conkey et al. demonstrated the use of Lee computer generated holograms ${ }^{18}$ to achieve beam focusing in scattering media with signal to background ratio up to $\sim 160$ in $\sim 34 \mathrm{~ms}$. An improved optical performance was achieved by projecting Lee holograms on the DMD along with spatial filtering and misaligned optical lenses for phase modulation using "Super-pixel" technique where each super-pixel is composed of multiple pixels of the $\mathrm{DMD}^{19}$. In $^{20}$, Drémeau et.al used the DMD as a binary input in a reference-less optical system for beam focusing. A Bayesian phase retrieval algorithm ${ }^{21}$ was used to precisely estimate the transmission matrix as well as for beam focusing through a white paint layer which acted as the highly scattering medium. In recent years ${ }^{22}$, Wang et.al implemented a binary-based digital optical phase conjugation (DOPC) system based on the use of DMD instead of SLMs. They used phase retrieval methods to design a binary computer generated hologram and demonstrated speed enhancement by approximately 2 orders of magnitude as compared to a liquid crystal SLM with a low playback latency of $\sim 5 \mathrm{~ms}$.

In display application, the DMD is used to modulate the time integrated light intensity through sequential pulse width modulation ${ }^{23-26}$. In the context of optical diffraction tomography ${ }^{27}$, Lee et.al demonstrated a timemultiplexing structured illumination control scheme using $\mathrm{DMDs}^{28}$. In their paper, the authors showed an improved performance by using time-multiplexed 8-bit amplitude modulation as compared to binary amplitude modulation (i.e. Lee holograms) in which the unwanted artifacts were diminished.

In this paper, we use a DMD to perform complex (amplitude and phase) modulation. We used the built-in dynamic range feature of the DMD device and to control the frame time of the DMD, to project an 8-bit image in $38.4 \mathrm{~ms}(256 \times 150 \mu \mathrm{s})$. We show that by splitting the complex field into real and imaginary parts and using the time modulation scheme of the DMD we were able to synthesize a complex signal. We demonstrated this method for wavefront shaping by phase conjugating a measured field in free space and/or passing through a scattering medium. This paves the way for imaging and optical phase conjugation systems that are fast, digital accuracy without compromising light efficiency. Finally, to increase further the frame rate for grayscale modulation using 


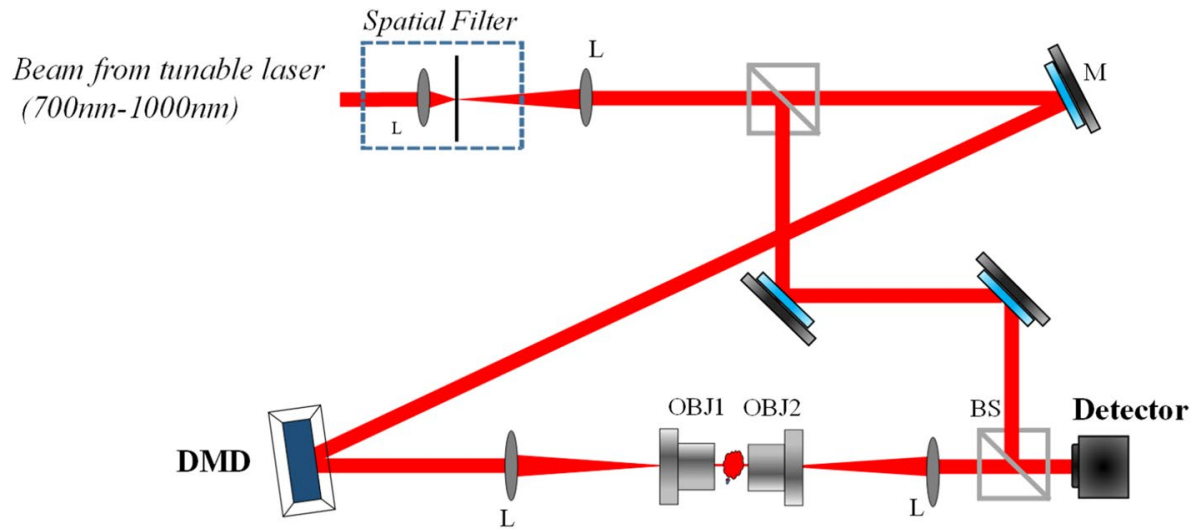

Figure 1. Optical setup. ( $M$ mirror, $L$ lens, $O B J$ objective lens, $B S$ beam splitter). 4 f systems are used to image DMD pattern onto the detector with a spatial magnification defined by the $4 \mathrm{f}$ systems.

the DMD, we modulated the intensity of the illuminating beam for each bit-plane while fixing the time for each bit-plane. Using this modulation scheme, the frame time for each grayscale image was reduced to $1.2 \mathrm{~ms}$ $(8 \times 150 \mu \mathrm{s})$.

In what follows, we first introduce the basic concept we use in the paper. After that, in the next sub-section in the methods section, we show how we used this technique for phase conjugation applications. We refer to this method as Opto-Electronic Phase Conjugation (OEPC) to distinguish it from conventional Digital Phase Conjugation. The results section was divided into 2 main sub-sections. The first subsection includes two experiments that demonstrate phase conjugation conducted using our time-multiplexed technique. The second sub-section shows a new modality for using the DMD which is amplitude modulation scheme.

\section{Methods}

Complex wavefront shaping with the DMD. We consider a complex field $f(x, y)=A(x, y) e^{j \varphi(x, y)}$ where $A(x, y)$ is the $2 \mathrm{D}$ amplitude and $\varphi(x, y)$ is the $2 \mathrm{D}$ phase pattern. We represent a complex pattern on the DMD by presenting positive and negative portions of real and imaginary parts of the complex field, sequentially. In order to represent the grayscale of the real and imaginary parts of the complex field, we take advantage of the time modulation feature of the DMD. $f(x, y)$ can be written as follows:

$$
\begin{aligned}
f(x, y) & =\operatorname{Re}\{f(x, y)\}+i * \operatorname{Im}\{f(x, y)\} \\
& =\sum_{\alpha=1}^{8} B R_{\alpha} 2^{\alpha-1}+i * \sum_{\alpha=1}^{8} B I_{\alpha} 2^{\alpha-1}
\end{aligned}
$$

where $B R_{\alpha}$ and $B I_{\alpha}$ are the $\alpha$ bit-planes for the real and imaginary parts, respectively. In our optical system, the field is interferometrically measured at the detector. This allows us to reconstitute the complete complex field by temporal integration on the detector which aggregates the contributions of each of the bit planes of both real and imaginary parts. Alternatively, the summation can be done post-detection on the computer.

We used the experimental setup shown in Fig. 1.

A continuous wave (CW) laser at $725 \mathrm{~nm}$ was used in all our experiments. The laser beam is spatially filtered and collimated with a pinhole and a pair of lenses. The signal beam is reflected from the DMD device (DLP4500NIR 0.45 WXGA near-Infrared, resolution: $912 \times 1140$, pixel pitch: $10.8 \mu \mathrm{m}$, Ajile Light Industries). A $4 \mathrm{f}$. system was used to image the DMD plane onto the detector plane to retrieve in-focus images. For holographic detection, the signal and reference beams are combined using a non-polarizing beam splitter and projected onto a complementary metal-oxide-semiconductor (CMOS) camera (Edmund optics, pixel size $=3.45 \mu \mathrm{m}$, resolution $=2056 \times 1542$ pixels). Although in the current experiment there is no object to be imaged, objective lenses were used to allow the DMD to be used for imaging applications.

The grayscale image was translated into 8 binary bit-planes (corresponding to the 8-bit image) and the 8 binary images were displayed onto the DMD sequentially to be captured onto the detector within a limited exposure time in a time-multiplexing manner. Since time-multiplexing is used, this means that the least significant bit-plane will be displayed for 1 time unit while the most significant bit-plane will be displayed for 128 time units (i.e. 1 time unit $=150 \mu \mathrm{s}$ ). This was repeated for both the real and the imaginary parts of the image. Figure 2 demonstrates successful phase modulation as a result of this technique. Each of the real and the imaginary parts were projected, one at a time, onto the DMD in a time multiplexing fashion. After capturing the two grayscale mages of the real and the imaginary parts, they were added digitally onto the computer which is equivalent to phase modulation by the DMD as seen in Fig. 2.

For phase maps whose phase exceeds $\pi / 2$ radians, we split the real and imaginary parts into positive and negative values and displayed each one separately onto the DMD. This is expressed by Eq. (2): 


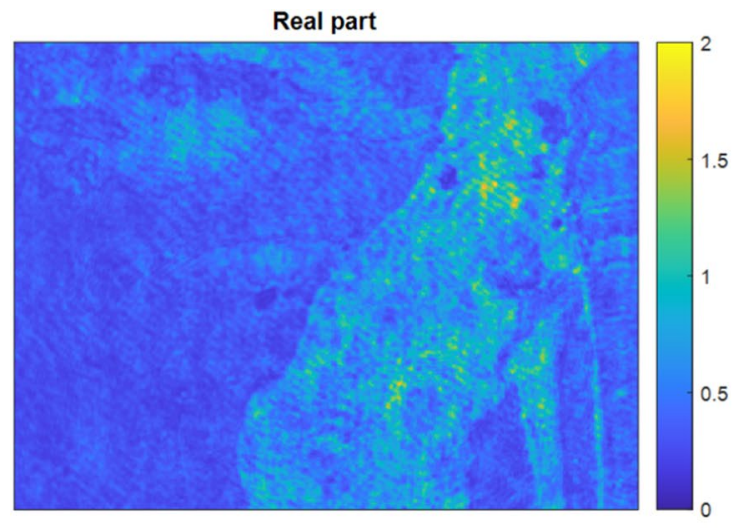

Magnitude

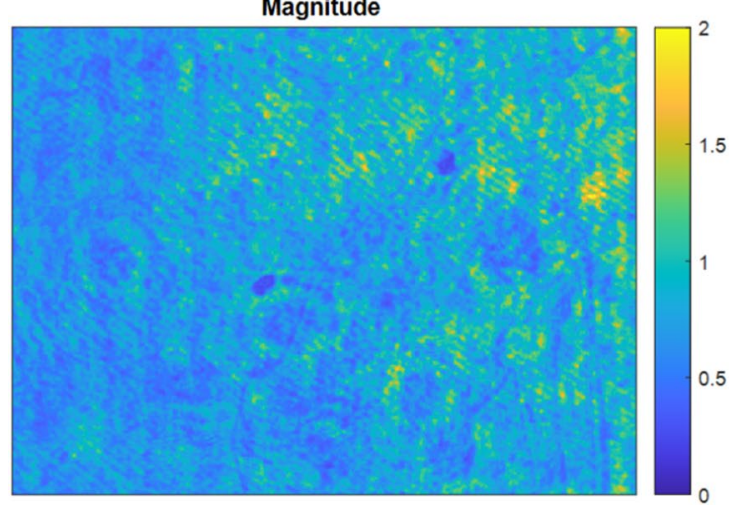

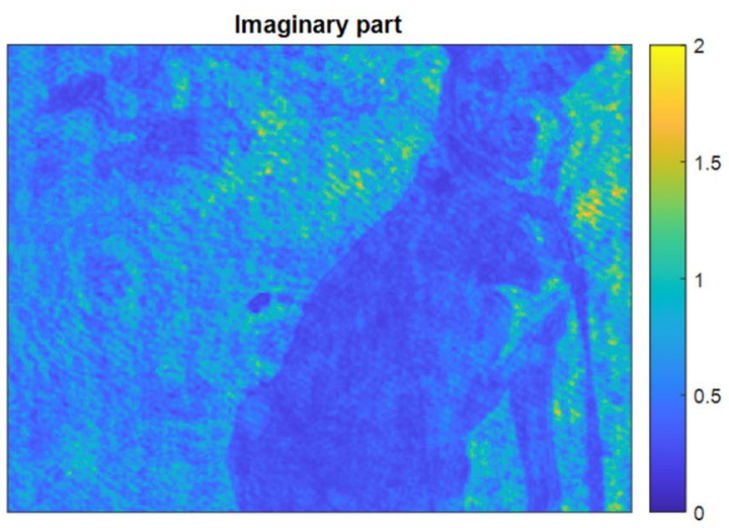

Phase

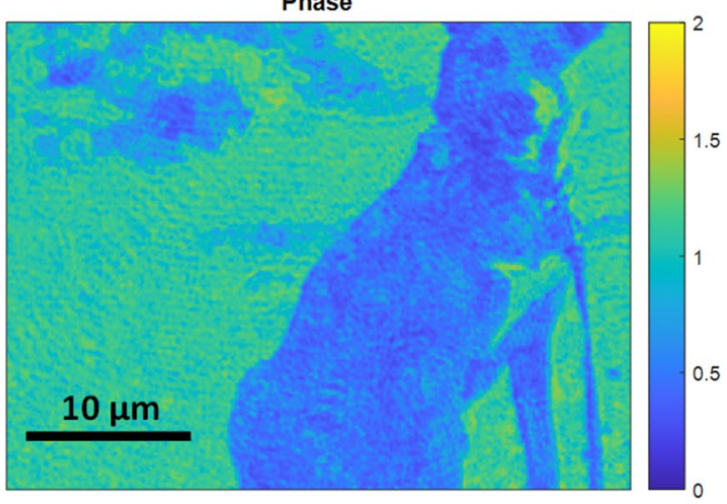

Figure 2. Phase modulation using DMD as expressed in equation (1) by splitting the complex field into real and imaginary parts.

$$
\begin{aligned}
f(x, y)= & \operatorname{Re}\{f(x, y)\}+i * \operatorname{Im}\{f(x, y)\} \\
= & {\left[\sum_{\alpha=1}^{8} B R_{\alpha}^{p} 2^{\alpha-1}-\sum_{\alpha=1}^{8} B R_{\alpha}^{n} 2^{\alpha-1}\right] } \\
& +i *\left[\sum_{\alpha=1}^{8} B I_{\alpha}^{p} 2^{\alpha-1}-\sum_{\alpha=1}^{8} B I_{\alpha}^{n} 2^{\alpha-1}\right]
\end{aligned}
$$

where $B R_{\alpha}^{p}$ and $B R_{\alpha}^{n}$ are the $\alpha$ bit-planes for the positive and negative parts of the real component, respectively, while $B I_{\alpha}^{p}$ and $B I_{\alpha}^{n}$ are the $\alpha$ bit-planes for the positive and negative parts of the imaginary component, respectively.

Opto-electronic phase conjugation (OEPC) using DMD. Assuming an initial complex field $\left(U_{i}\right)$ incident on a scattering medium, we can write the output as follows:

$$
U_{o}=T_{i o} U_{i}
$$

where $T_{i o}$ is the transmission matrix describing the propagation of $U_{i}$ through the scattering medium. After taking the complex conjugate of this function and propagate through the same medium, we get:

$$
\begin{aligned}
U_{\text {final }} & =T_{o i} U_{o}^{*} \\
& =T_{o i}\left(T_{i o} U_{i}\right)^{*}=T_{o i} T_{i o}^{*} U_{i}^{*}
\end{aligned}
$$

Assuming a perfect time-symmetric system (i.e. $T_{o i}^{-1}=T_{i o}^{*}$ ), Eq. 4 simplifies to:

$$
U_{\text {final }}=T_{o i} T_{i o}^{*} U_{i}^{*}=T_{o i} T_{o i}^{-1} U_{i}^{*}=U_{i}^{*}
$$

Equation (5) shows how OEPC removes the aberrations associated with the medium (i.e. free space propagation or mouse liver tissue in our experiments) and the input field is retrieved. In order to demonstrate OEPC using the DMD, a simplified interferometric setup was used as shown in Fig. 3. The detector used in this experiment is a CMOS camera (Photonfocus Inc., resolution: $1312 \times 1280$, pixel size: $8 \mu \mathrm{m}$ ). First, the setup was calibrated to ensure pixel-to-pixel alignment between the DMD and the camera. The incident angle is chosen to be at $\theta$ degrees with respect to the direction normal to the DMD surface. Given that the DMD has an "on" and "off" state beam at $\pm 12^{\circ}$ with respect to the normal direction, $\theta$ was chosen to be around $12^{\circ}$ relative to the normal direction as shown in Fig. 3b. Owing to the specific distribution of the pixels in the DMD, (Fig. 3a), 

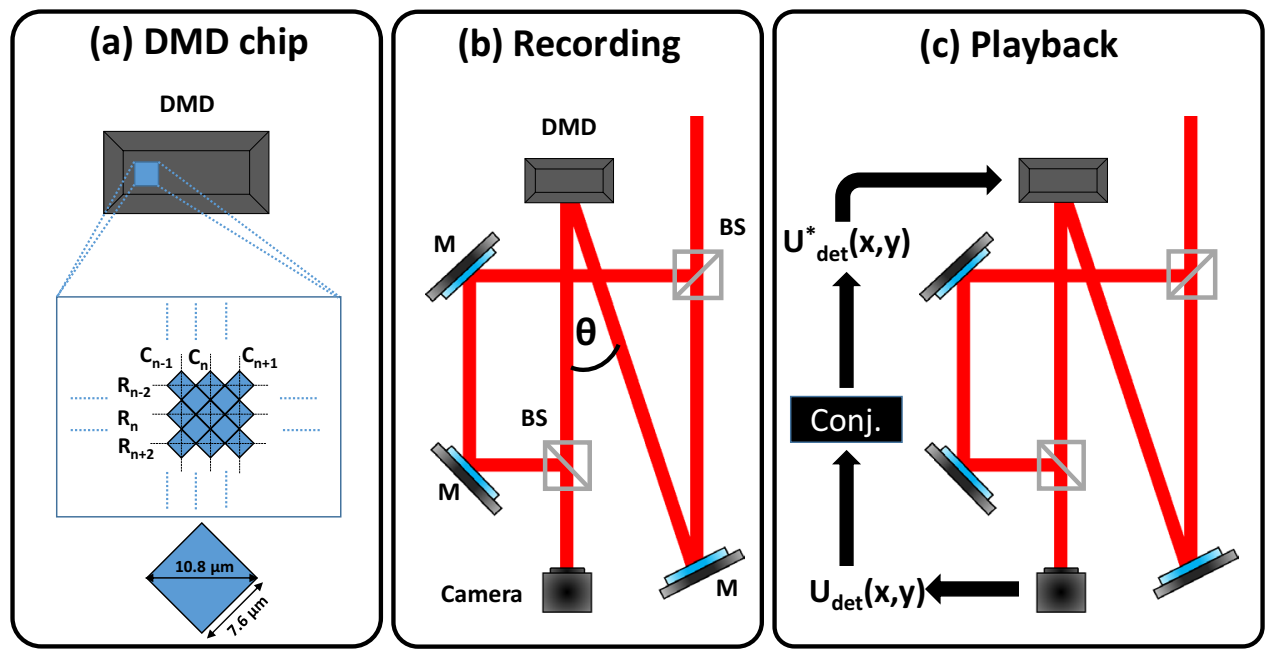

Figure 3. OEPC optical setup. (a) DMD projector grid showing the pixel orientation. (b) Recording step is performed first to record the hologram after free-space propagation. The second step is the (c) playback where the complex conjugate of the detected field is displayed onto the DMD and the original field is retrieved. $M$ mirror, $B S$ beamsplitter.

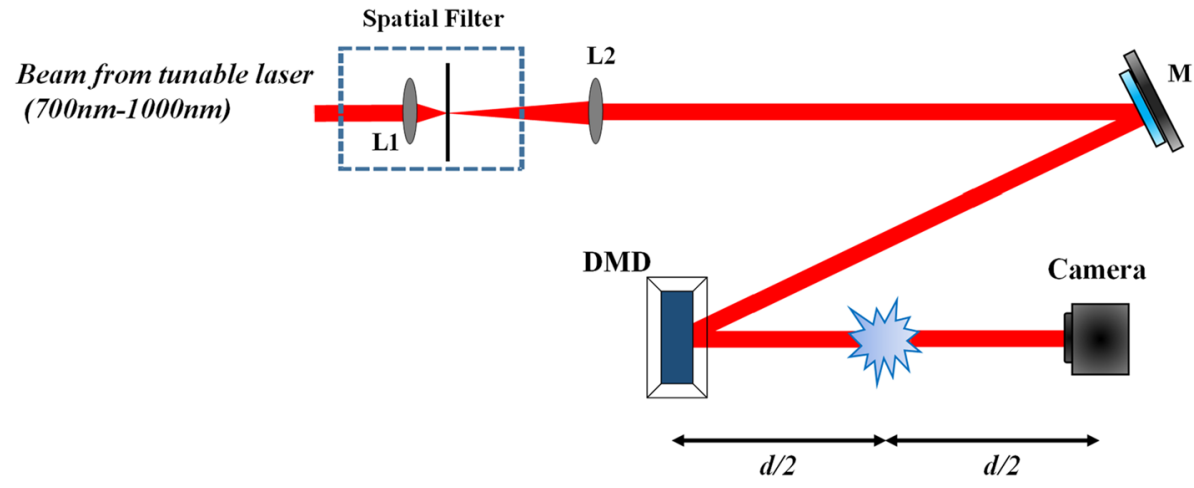

Figure 4. Experimental setup with the scattering medium inserted in between the DMD and the camera $(M$ mirror, $L 1 \nLeftarrow L 2$ lenses, $\mathrm{d}=28 \mathrm{~cm}$ ).

interpolation was used in order to successfully align the pixels of the DMD and SLM. As seen in Fig. 3a, the center-to-center distance between two successive columns (i.e. $C_{n-1}$ and $C_{n}$ ) is $10.8 \mu \mathrm{m}$ while the distance between two successive rows is equal to $5.4 \mu \mathrm{m}$ (i.e. $10.8 / 2 \mu \mathrm{m}$ ) as given by the DMD specification. This difference was accommodated by the use of interpolation to ensure successful mapping of the detector image onto the DMD screen. After calibrating the system, the recording step (Fig. 3b) was performed to by displaying phase and/or amplitude maps as expressed by Eq. (2). After acquiring the image on the CMOS detector, the hologram was processed and the complex field was extracted on a personal computer and its complex conjugate was displayed back onto the DMD (Fig. 3c) and finally the original non-distorted image is reconstructed by adding the real and the imaginary parts digitally.

\section{Results}

OEPC for diffracted pattern after passing through scattering media. An experiment was performed with a scattering medium in between the DMD and the detector as shown in Fig. 4 . The scattering medium was a $10 \mu \mathrm{m}$-thick fixed mouse liver-tissue sandwiched between a standard cover-slide of $1 \mathrm{~mm}$ thickness and a coverslip of $170 \mu \mathrm{m}$ thickness.

In the OEPC experiment, a simple object (a binary amplitude circular mask of $\sim 430 \mu \mathrm{m}$ diameter) was displayed on the DMD and the reflected light was captured by the detector. By extracting the complex field of the acquired hologram, taking its complex conjugate and feeding it back to the DMD with the scattering medium in place, we were able to retrieve the original pattern. Figure 5 shows the diffracted and the refocused spot after OEPC. Figure $5 \mathrm{~b}$ shows how we get a full-width at half-maximum (FWHM) of $250 \mu \mathrm{m}$ whereas the defocused spot (Fig. 5a) has a FWHM of $400 \mu \mathrm{m}$. 
(a) Diffracted pattern

Normalized Intensity

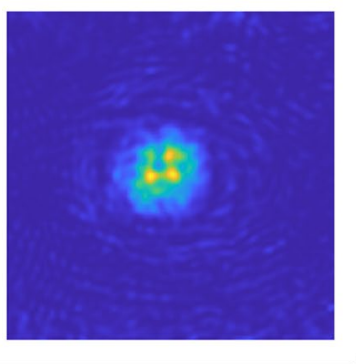

\section{(b) Refocused pattern}

Normalized Intensity

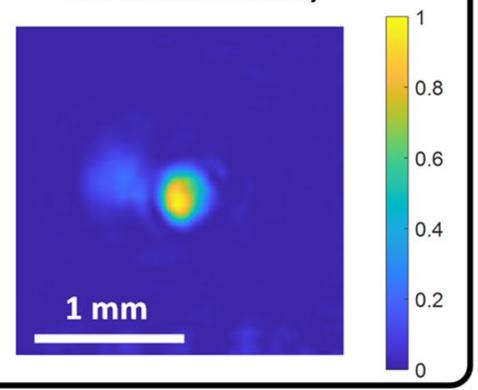

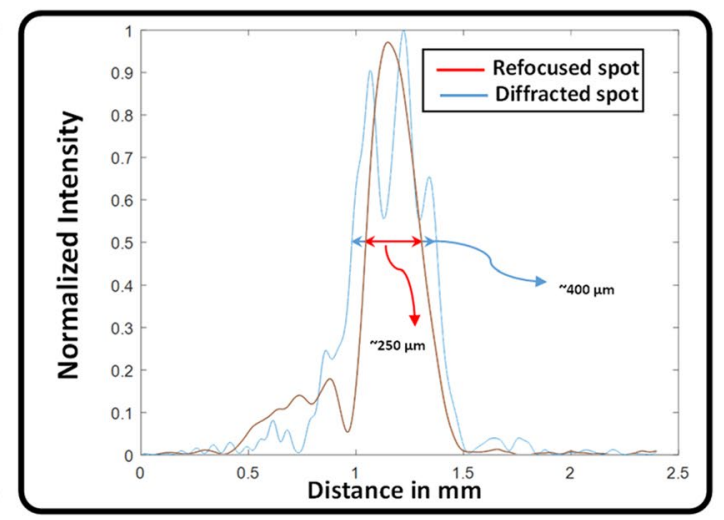

Figure 5. Intensity pattern before and after refocusing using OEPC technique. (a) Diffracted intensity pattern after propagating through the scattering medium path while (b) shows the intensity pattern after OEPC.

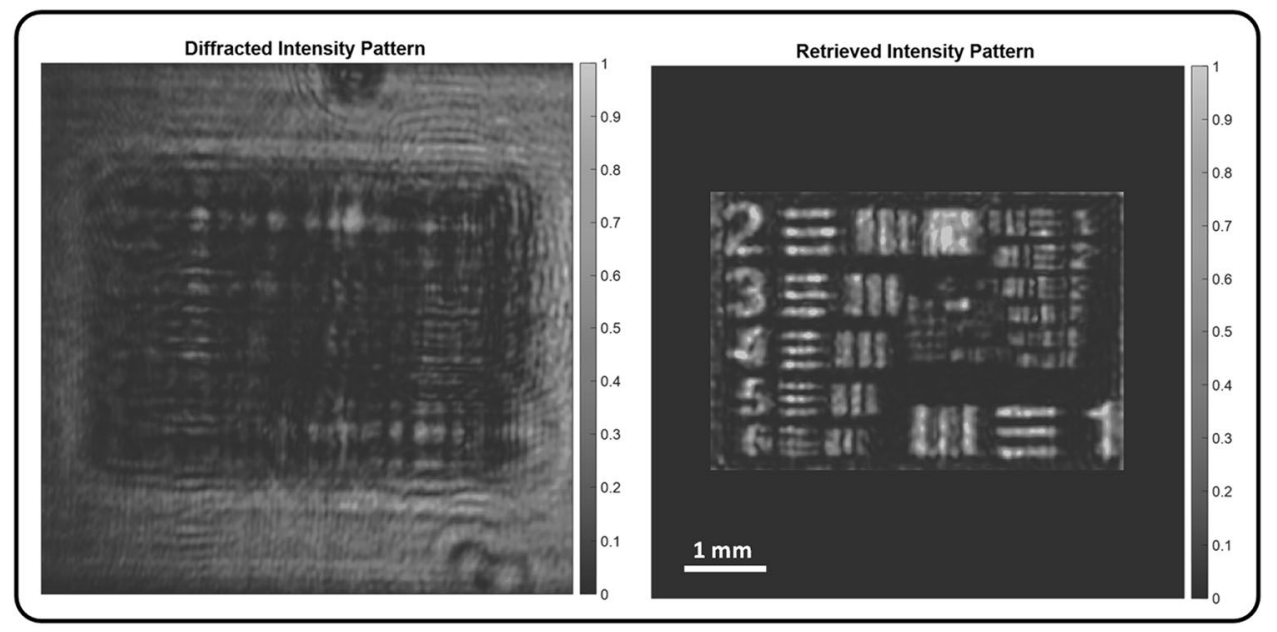

Figure 6. Intensity pattern before and after refocusing using OEPC. Left: diffracted intensity pattern after propagating through the scattering medium. Right: shows the intensity pattern after OEPC.

A similar experiment was performed with the USAF target being the input amplitude-only field passing through the same scattering medium (i.e. liver tissue). The image on the left hand-side of Fig. 6 shows the diffracted intensity pattern through the sample while the right hand-side image shows the refocused USAF target after compensating the effect of defocusing and scattering through the sample.

Grayscale modulation using amplitude modulator synchronized with the DMD. In order to boost further the speed of grayscale modulation using the DMD, amplitude modulation was used instead of time modulation. As shown in Fig. 7, an electro-optic modulator (Thorlabs, EO-AM-NR-C1) was used to modulate the light intensity for each bit-plane. In this case we used light intensity instead of light amplitude since holographic recording was not used on the camera. Therefore the calibration was done for intensity recording. The NI PCIe-6321 data acquisition tool was used for fast synchronization permitting speeds up to $90 \mathrm{kHz}$ which is much faster than the DMD speed $(6.6 \mathrm{kHz})$. Figure 7 shows the calibration process of the amplitude modulator (AM). A photodetector (Thorlabs, PDA36A-EC) was used to measure the voltage signal from the AM as a function of the applied voltage. As the characteristic half-wave voltage $\left(V_{\pi}\right)$ at $725 \mathrm{~nm}$ for the AM was around $240 \mathrm{~V}$, high voltage was needed to get the full modulation range which was generated using a high voltage amplifier (HVA200, $\mathrm{V}_{\text {in }}=-10: 10, \mathrm{~V}_{\text {out }}=-200: 200$, Thorlabs). A quarter wave plate (QWP) was used to get the full range since $V_{\pi}$ is higher than the maximum possible voltage generated by the voltage amplifier.

A look-up-table (LUT) is extracted from Fig. 7c to modulate the 8 bit-planes of the 8-bit grayscale image. After calibrating the AM, the amplitude modulator was synchronized with the DMD and images were recorded using the camera as shown in Fig. 8. With such modulation scheme a maximum achievable frame rate of $833 \mathrm{~Hz}$ could be reached (i.e. $\frac{1}{150 \mu \mathrm{s} \times 8}$ ). Using this amplitude-modulation scheme, we showed efficient grayscale modulation for sinusoidal grating using the DMD (see supplementary video).

Figure 9 shows an example of amplitude modulation for a sinusoidal grating with a grating period of 15 pixels displayed onto the DMD and imaged by the camera where it shows the effectiveness of our proposed scheme 


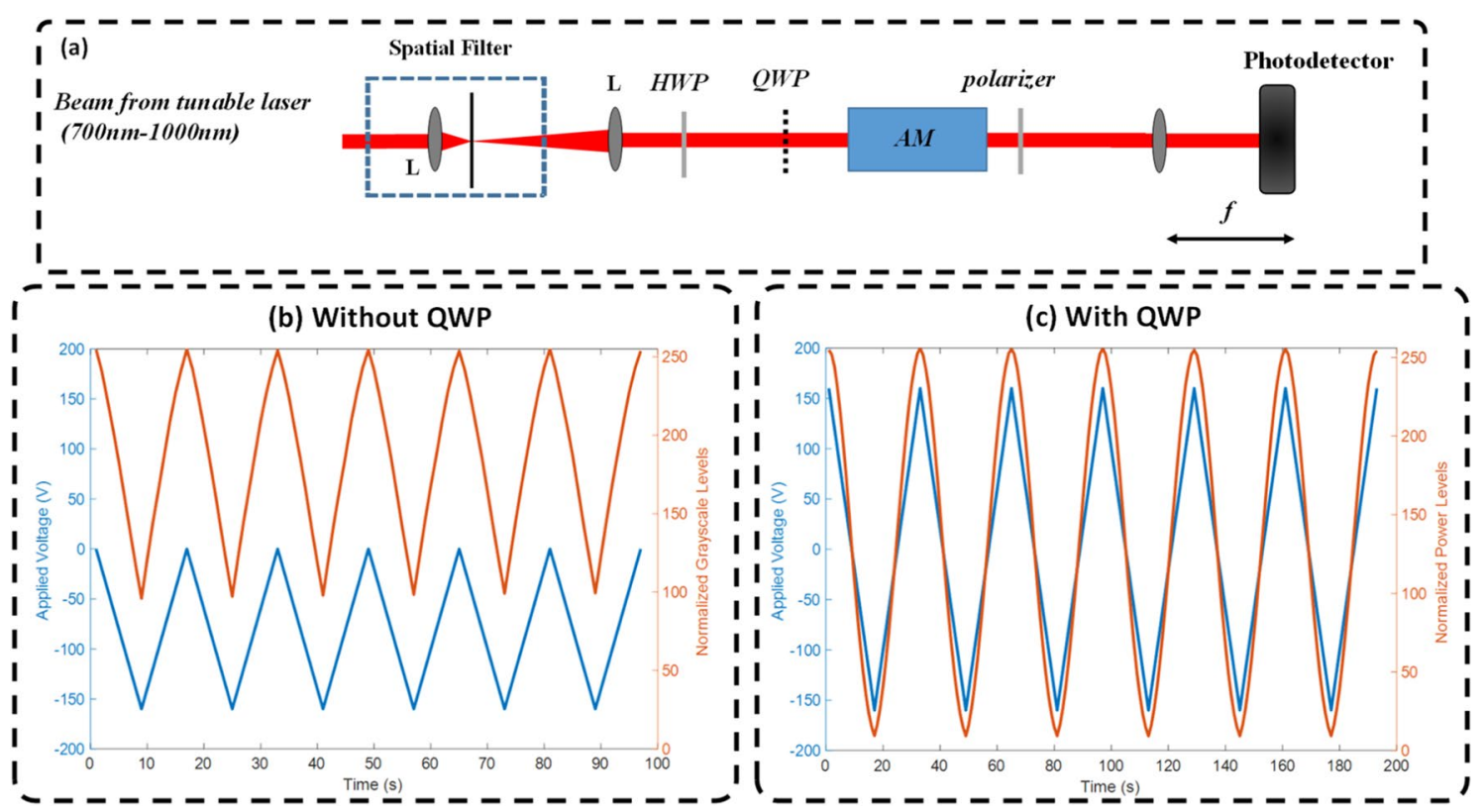

Figure 7. Amplitude modulation. (a) Calibration system for the amplitude modulator. (b) Amplitude modulation (orange) as a function of the applied voltage (blue) without the QWP. (c) Amplitude modulation (orange) as a function of the applied voltage (blue) with the QWP. Comparing (b) and (c), adding the QWP resulted in creating an input circular polarization which resulted in getting the complete modulation range.

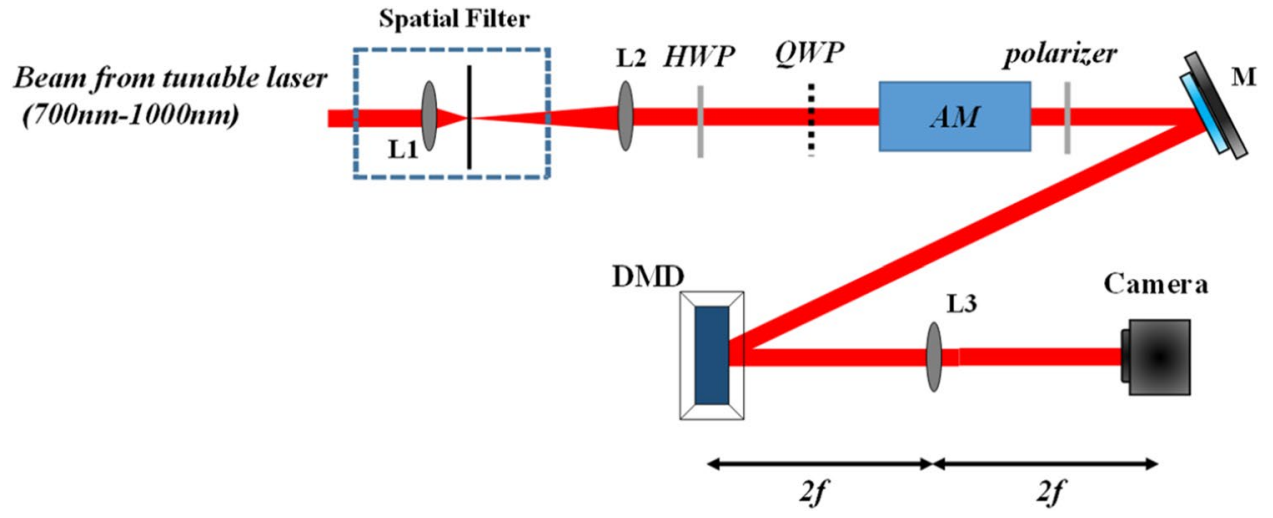

Figure 8. Amplitude modulation using AM synchronized with the DMD.

as compared to binary grating where the higher diffraction orders are visible as shown in Fig. 9(b1). For the amplitude modulation case, higher orders are highly suppressed while the 0 th $,+1,-1$ orders are dominant.

The DMD consists of individually-controlled mirrors, and this creates many diffraction orders ${ }^{29}$. This phenomenon is shown in Fig. 10 where the pattern recorded on the DMD (a square) is repeated multiple times. The photograph in Fig. 10 was taken by placing the camera close to the DMD in order to capture the multiple patterns and demonstrate the effect.

During the performance of the experiment it was noticed that using a lens (L3 in Fig. 8) with higher focal length showed better reconstructed images. This is due to the fact that as the lens gets far from the DMD, additional diffracted orders from the DMD miss the lens and are not captured by the camera while only the zero-diffracted beam is able to pass through the lens and be captured by the camera. Notice that in practice, this approach is not light efficient, and a lens design such as the one used in display applications ${ }^{30,31}$ that captures all the diffracted orders and superimposes them on the camera is preferable.

To further validate our results, phase modulation was performed as described earlier in which the complex field is divided into real and imaginary components and then each component was presented separately using the electro-optic amplitude modulation scheme. We recorded directly intensity on the camera where the signals corresponding to the different bit planes were integrated on the camera. The real and imaginary parts were readout and were added digitally on the pc to form the complex field. A blazed grating and a binary phase grating were synthesized on the DMD. As shown in Fig. 11, the blazed grating shows all the power focused into the +1 order. On the other hand, the binary phase grating resulted in multiple orders as expected. 

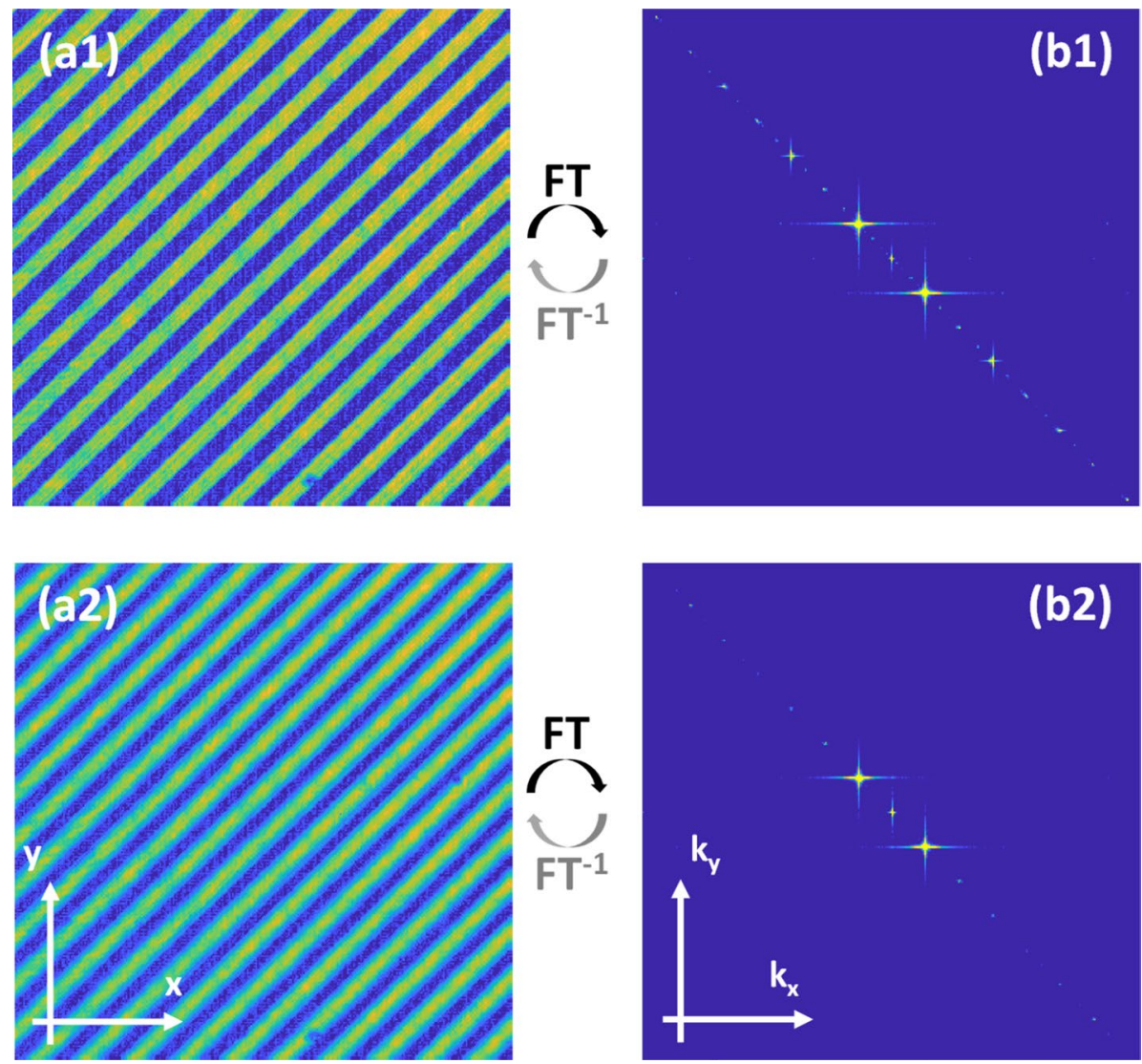

Figure 9. Sinusoidal grating projected from the DMD using amplitude modulation scheme. (a1, a2) The recorded gratings. (b1, b2) Fourier transform of recorded gratings. $(\mathbf{a 1}, \mathbf{b 1})$ show the binary grating and the associated Fourier transform where the diffraction noise (artifacts) are dominant. (a2, b2) show the sinusoidal grating and the associated Fourier transform in which the 3 main orders are visible with no visible diffraction artifacts.

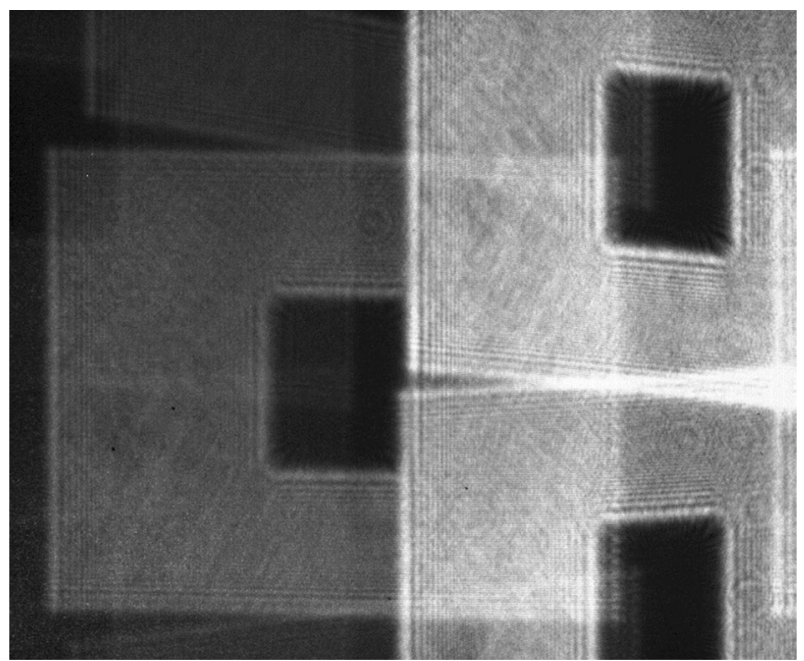

Figure 10. Diffraction patterns from the DMD as a result of mirror deflections. 


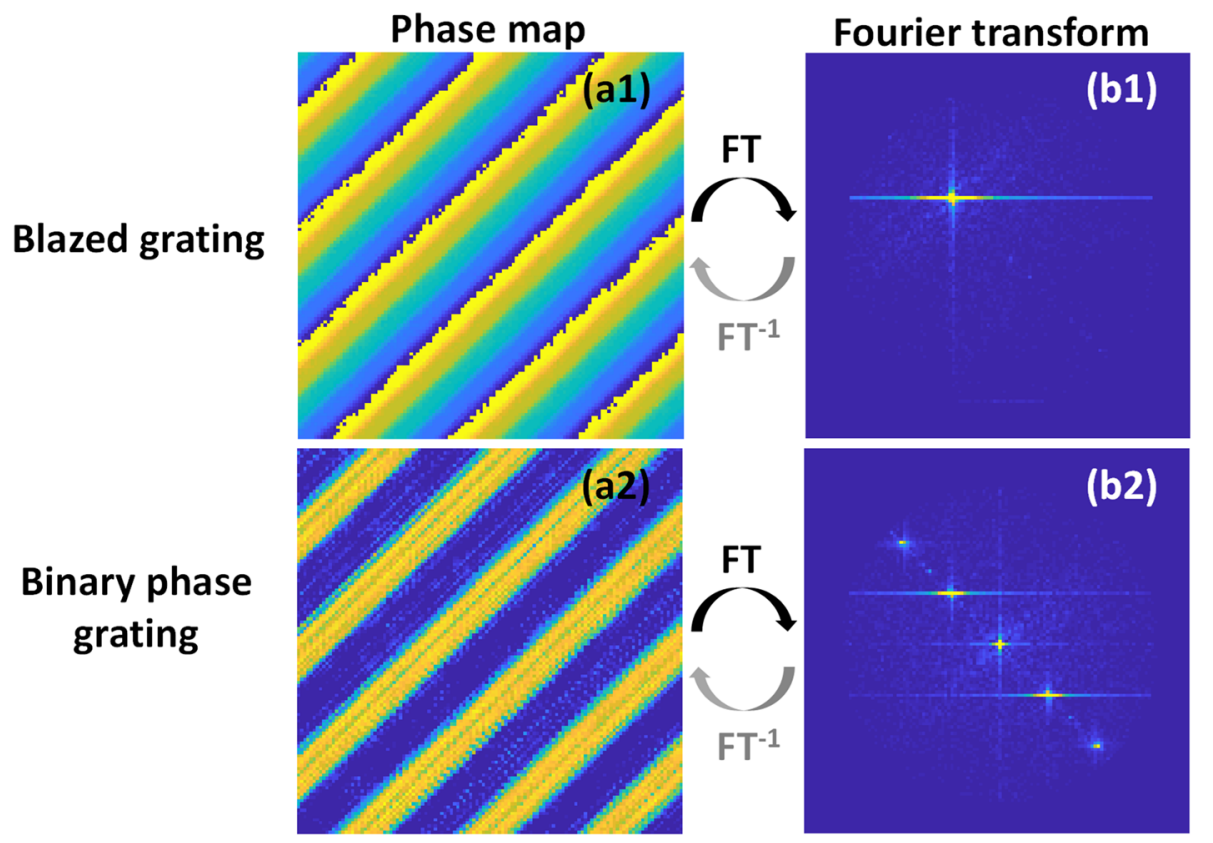

Figure 11. Phase gratings projected from the DMD using amplitude modulation scheme. (a1, a2) The recorded gratings. (b1, b2) Fourier transform of recorded gratings. (a1, b1) show the blazed grating and the associated Fourier transform where the diffraction noise (artifacts) are dominant. (a2, b2) show the binary phase grating and the associated Fourier transform in which the multiple orders are present.

(a) 2-bit

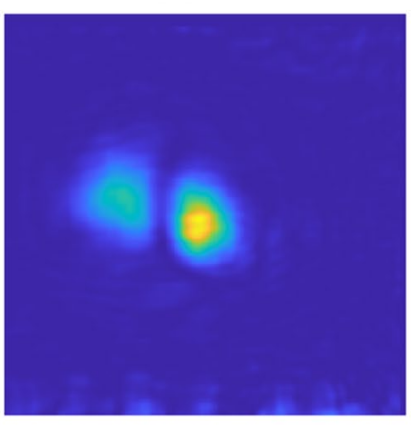

(b) 4-bit

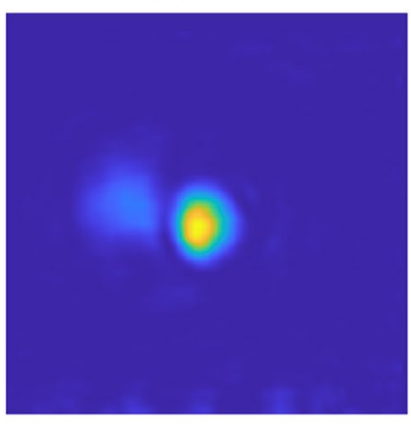

(c) 8-bit

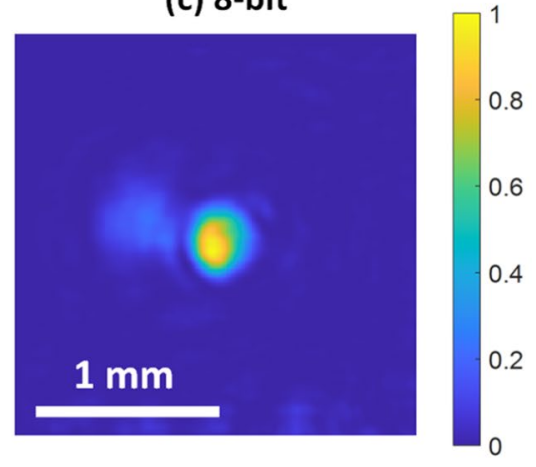

Figure 12. Effect of changing the modulation depth on the focusing quality. (a) 2-bit, (b) 4-bit, (c) 8-bit. (b), and (c) show well preserved focused spot while (a) shows stronger artifacts.

\section{Discussion}

In the optical OEPC experiments, the curvature of the DMD surface was corrected digitally by acquiring another hologram with all the DMD pixels in the "on" state (i.e. background hologram), then all the collected holograms for the real and the imaginary parts were corrected by dividing the extracted fields over the background field, this resulted in a clean image without phase-artifacts as shown in Figs. 2, 3, 4, 5, and 6. For the time-multiplexing scheme, the 8-bit frame rate is rather low ( 26 frames per second). This speed can be further boosted using a faster commercially available $\mathrm{DMD}^{32}$ (refresh rates $\sim 32 \mathrm{kHz}$ instead of $6.6 \mathrm{kHz}$ ) and also reducing the region of interest (ROI) on the DMD if the application allows a smaller number of pixels. For instance, using the same DMD we used in our study, by decreasing the ROI to $16 \times 1140$ instead of $912 \times 1140$, refresh rates can be boosted to $100 \mathrm{kHz}$ (corresponding to a complex-field display rate $\sim 110$ frames per second). The speed of time modulation technique can be further enhanced by using 4-bit images instead of 8-bit images which will yield a speed enhancement by a factor of 16 . Figure 12 shows the effect of changing the number of bits used in the DMD when carrying out OEPC on the focusing quality after passing through the scattering medium (the liver tissue). It is observed that both 4-bit (Fig. 12b) and 8-bit (Fig. 12c) modulation depth resulted in a well preserved focused spot however with a speed enhancement factor of 16 with a display time of $2.4 \mathrm{~ms}(2.4 \mathrm{~ms}=150 \mu \mathrm{s} \times 16)$ with an effective frame rate of 415 frames per second. 


\begin{tabular}{|l|l|l|}
\hline Modulationlspecs & Frame rate & Minimum display time \\
\hline Binary (1-bit) & $6.6 \mathrm{kHz}$ & $150 \mu \mathrm{s}$ \\
\hline Time (8-bit) & $26 \mathrm{~Hz}=\frac{6.66 \mathrm{kHz}}{2^{8}}$ & $38.4 \mathrm{~ms}=150 \mu \mathrm{s} \times 2^{8}$ \\
\hline Time (4-bit) & $416 \mathrm{~Hz}=\frac{6.66 \mathrm{kHz}}{2^{4}}$ & $2.4 \mathrm{~ms}=150 \mu \mathrm{s} \times 2^{4}$ \\
\hline Amplitude (8-bit) & $833 \mathrm{~Hz}=\frac{6.66 \mathrm{kHz}}{8}$ & $1.2 \mathrm{~ms}=150 \mu \mathrm{s} \times 8$ \\
\hline Amplitude (4-bit) & $1665 \mathrm{~Hz}=\frac{6.66 \mathrm{kHz}}{4}$ & $0.6 \mathrm{~ms}=150 \mu \mathrm{s} \times 4$ \\
\hline
\end{tabular}

Table 1. Comparison between different modulation schemes in terms of frame rate/display time.

\begin{tabular}{|l|l|l|}
\hline Modulation $\backslash$ specs & Frame rate & Minimum display time \\
\hline Binary (1-bit) & $32 \mathrm{kHz}$ & $31.25 \mu \mathrm{s}$ \\
\hline Time (8-bit) & $125 \mathrm{~Hz}=\frac{32 \mathrm{kHz}}{2^{8}}$ & $8 \mathrm{~ms}=31.25 \mu \mathrm{s} \times 2^{8}$ \\
\hline Time (4-bit) & $2 \mathrm{kHz}=\frac{32 \mathrm{kHz}}{2^{4}}$ & $0.5 \mathrm{~ms}=31.25 \mu \mathrm{s} \times 2^{4}$ \\
\hline Amplitude (8-bit) & $4 \mathrm{kHz}=\frac{32 \mathrm{kHz}}{8}$ & $250 \mu \mathrm{s}=31.25 \mu \mathrm{s} \times 8$ \\
\hline Amplitude (4-bit) & $8 \mathrm{kHz}=\frac{32 \mathrm{kHz}}{4}$ & $125 \mu \mathrm{s}=31.25 \mu \mathrm{s} \times 4$ \\
\hline
\end{tabular}

Table 2. Comparison between different modulation schemes in terms of frame rate/display time for DMD with refresh rate of $32 \mathrm{kHz}$.

Table 1 shows a comparison between amplitude and time modulation schemes in terms of the frame rate for the used DMD whose refresh rate is $6.66 \mathrm{kHz}$.

Current DMD technologies have refresh rate as high as $32 \mathrm{kHz}$ which would scale up the modulation speed as shown in Table 2.

\section{Conclusion}

High speed, complex wavefront shaping using the digital micro-mirror device was demonstrated. The DMD was used in a time-multiplexing mode which allows for 8-bit grayscale image representation on the DMD within $38.4 \mathrm{~ms}$ allowing for focusing in live samples or for other applications without compromising the accuracy. For higher modulation rates, amplitude modulation was demonstrated with maximum achievable frame rate for 8-bit images of $833 \mathrm{~Hz}$ yielding a speed up by a factor of 32 as compared to time multiplexing schemes.

The method we demonstrated relies on time integrating holographic recordings of the fields or intensity patterns corresponding to multiple binary patterns on the DMD. The method is particularly useful for optical systems that use digital holography. The method might also be useful in applications where the projected pattern is integrated in time by a light sensitive material such as a fluorescent molecule in imaging or a photo-initiator molecule in $3 \mathrm{D}$ printing.

Received: 7 July 2021; Accepted: 7 September 2021

Published online: 22 September 2021

\section{References}

1. Vellekoop, I. M., Lagendijk, A. \& Mosk, A. P. Exploiting disorder for perfect focusing. Nat. Photonics 4, 320-322 (2010).

2. Yilmaz, H., Vos, W. L. \& Mosk, A. P. Optimal control of light propagation through multiple-scattering media in the presence of noise. Biomed. Opt. Express 4, 1759-1768 (2013).

3. Popoff, S., Lerosey, G., Fink, M., Boccara, A. C. \& Gigan, S. Image transmission through an opaque material. Nat. Commun. 1, 81 (2010).

4. Chaigne, T. et al. Controlling light in scattering media noninvasively using the photoacoustic transmission matrix. Nat. Photonics 8, 58-64 (2014).

5. Yoon, J., Lee, K., Park, J. \& Park, Y. Measuring optical transmission matrices by wavefront shaping. Opt. Express 23, 10158-10167 (2015).

6. Yaqoob, Z., Psaltis, D., Feld, M. S. \& Yang, C. Optical phase conjugation for turbidity suppression in biological samples. Nat. Photonics 2, 110-115 (2008).

7. Papadopoulos, I. N., Farahi, S., Moser, C. \& Psaltis, D. Focusing and scanning light through a multimode optical fiber using digital phase conjugation. Opt. Express 20, 10583-10590 (2012).

8. Hsieh, C.-L., Pu, Y., Grange, R. \& Psaltis, D. Digital phase conjugation of second harmonic radiation emitted by nanoparticles in turbid media. Opt. Express 18, 12283-12290 (2010).

9. Ren, Y.-X., Lu, R.-D. \& Gong, L. Tailoring light with a digital micromirror device. Ann. Phys. 527, 447-470 (2015).

10. Akbulut, D., Huisman, T. J., van Putten, E. G., Vos, W. L. \& Mosk, A. P. Focusing light through random photonic media by binary amplitude modulation. Opt. Express 19, 4017-4029 (2011).

11. Nam, K. \& Park, J.-H. Increasing the enhancement factor for DMD-based wavefront shaping. Opt. Lett. 45, 3381-3384 (2020).

12. Turpin, A., Vishniakou, I. \& Seelig, J. D. Light scattering control in transmission and reflection with neural networks. Opt. Express 26, 30911-30929 (2018).

13. Zhao, T., Ourselin, S., Vercauteren, T. \& Xia, W. Focusing light through multimode fibres using a digital micromirror device: A comparison study of non-holographic approaches. Opt. Express 29, 14269-14281 (2021). 
14. Shin, S., Kim, K., Yoon, J. \& Park, Y. K. Active illumination using a digital micromirror device for quantitative phase imaging. Opt. Lett. 40, 5407-5410 (2015).

15. Bianchi, S. \& Di Leonardo, R. A multi-mode fiber probe for holographic micromanipulation and microscopy. Lab Chip 12(3), 635-639 (2012).

16. Turtaev, S. et al. Comparison of nematic liquid-crystal and DMD based spatial light modulation in complex photonics. Opt. Express 25, 29874-29884 (2017).

17. Conkey, D. B., Caravaca-Aguirre, A. M. \& Piestun, R. High-speed scattering medium characterization with application to focusing light through turbid media. Opt. Express 20, 1733-1740 (2012).

18. Lee, W.-H. Binary computer-generated holograms. Appl. Opt. 18, 3661-3669 (1979).

19. Goorden, S. A., Bertolotti, J. \& Mosk, A. P. Superpixel-based spatial amplitude and phase modulation using a digital micromirror device. Opt. Express 22, 17999-18009 (2014).

20. Drémeau, A. et al. Reference-less measurement of the transmission matrix of a highly scattering material using a DMD and phase retrieval techniques. Opt. Express 23, 11898-11911 (2015).

21. Drémeau, A. \& Krzakala, F. Phase recovery from a Bayesian point of view: The variational approach. In Proceedings of IEEE Transactions on Acoustics, Speech, and Signal Processing (2015).

22. Wang, D. et al. Focusing through dynamic tissue with millisecond digital optical phase conjugation. Optica 2, 728-735 (2015).

23. Ren, Y.-X. et al. Experimental generation of Laguerre-Gaussian beam using digital micromirror device. Appl. Opt. 49, 1838-1844 (2010).

24. Gong, L. et al. Generation of nondiffracting Bessel beam using digital micromirror device. Appl. Opt. 52, 4566-4575 (2013).

25. Adeyemi, A. A., Barakat, N. \& Darcie, T. E. Applications of digital micro-mirror devices to digital optical microscope dynamic range enhancement. Opt. Express 17, 1831-1843 (2009).

26. Ding, X., Ren, Y. \& Lu, R. Shaping super-Gaussian beam through digital micro-mirror device. Sci. China Phys. Mech. Astron. 58, $1-6(2015)$.

27. Jin, D., Zhou, R., Yaqoob, Z. \& So, P. T. C. Tomographic phase microscopy: Principles and applications in bioimaging. J. Opt. Soc. Am. B 34, B64-B77 (2017).

28. Lee, K., Kim, K., Kim, G., Shin, S. \& Park, Y. K. Time-multiplexed structured illumination using a DMD for optical diffraction tomography. Opt. Lett. 42, 999-1002 (2017).

29. Park, M.-C., Lee, B.-R., Son, J.-Y. \& Chernyshov, O. Properties of DMDs for holographic displays. J. Mod. Opt. 62(19), 1600-1607. https://doi.org/10.1080/09500340.2015.1054445 (2015)

30. Pan, J.-W. \& Wang, H.-H. High contrast ratio prism design in a mini projector. Appl. Opt. 52, 8347-8354 (2013).

31. Pan, J.-W., Wang, C.-M., Sun, W.-S. \& Chang, J.-Y. Portable digital micromirror device projector using a prism. Appl. Opt. 46, 5097-5102 (2007).

32. Texas Instruments Incorporated. DLP7000. https://www.ti.com/product/DLP7000.

\section{Author contributions}

A.B.A. and D.P. conceived the experiments, A.B.A. conducted the experiments. All authors reviewed the manuscript.

\section{Funding}

Innosuisse-Schweizerische Agentur für Innovationsförderung (34247.1 IP-ENG) and Swiss National Science Foundation (SNSF 514481)

\section{Competing interests}

The authors declare no competing interests.

\section{Additional information}

Supplementary Information The online version contains supplementary material available at https://doi.org/ 10.1038/s41598-021-98430-w.

Correspondence and requests for materials should be addressed to A.B.A.

Reprints and permissions information is available at www.nature.com/reprints.

Publisher's note Springer Nature remains neutral with regard to jurisdictional claims in published maps and institutional affiliations.

(c) Open Access This article is licensed under a Creative Commons Attribution 4.0 International License, which permits use, sharing, adaptation, distribution and reproduction in any medium or format, as long as you give appropriate credit to the original author(s) and the source, provide a link to the Creative Commons licence, and indicate if changes were made. The images or other third party material in this article are included in the article's Creative Commons licence, unless indicated otherwise in a credit line to the material. If material is not included in the article's Creative Commons licence and your intended use is not permitted by statutory regulation or exceeds the permitted use, you will need to obtain permission directly from the copyright holder. To view a copy of this licence, visit http://creativecommons.org/licenses/by/4.0/.

(c) The Author(s) 2021 